\title{
Origins of spontaneous activity in the degenerating retina
}

\author{
Stuart Trenholm ${ }^{1 *}$ and Gautam B. Awatramani ${ }^{2}$ \\ ${ }^{1}$ Friedrich Miescher Institute for Biomedical Research, Basel, Switzerland, ${ }^{2}$ Department of Biology, University of Victoria, \\ Victoria, BC, Canada
}

Sensory deafferentation resulting from the loss of photoreceptors during retinal degeneration ( $\mathrm{rd}$ ) is often accompanied by a paradoxical increase in spontaneous activity throughout the visual system. Oscillatory discharges are apparent in retinal ganglion cells in several rodent models of rd, indicating that spontaneous activity can originate in the retina. Understanding the biophysical mechanisms underlying spontaneous retinal activity is interesting for two main reasons. First, it could lead to strategies that reduce spontaneous retinal activity, which could improve the performance of vision restoration strategies that aim to stimulate remnant retinal circuits in blind patients. Second, studying emergent network activity could offer general insights into how sensory systems remodel upon deafferentation. Here we provide an overview of the work describing spontaneous activity in the degenerating retina, and outline the current state of knowledge regarding the cellular and biophysical properties underlying spontaneous neural activity.

OPEN ACCESS

Edited by:

Steven F. Stasheff,

University of lowa, USA

Reviewed by:

Rafael Linden,

Federal University of Rio de Janeiro,

Brazil

Botir T. Sagdullaev,

Weill Cornell Medical College, USA

*Correspondence:

Stuart Trenholm

Friedrich Miescher Institute for Biomedical Research

Maulbeerstrasse 66, 4058 Basel,

Switzerland

stuart.trenholm@fmi.ch

Received: 15 May 2015 Accepted: 06 July 2015 Published: 29 July 2015

Citation:

Trenholm S and Awatramani GB (2015) Origins of spontaneous activity

in the degenerating retina.

Front. Cell. Neurosci. 9:277.

doi: 10.3389/fncel.2015.00277
Keywords: retinal degeneration, oscillations, All amacrine cells, gap junctions, $\mathrm{Na}^{+}$channels, retina, bipolar cells, ganglion cells

\section{The Degenerating Retina is Intrinsically Noisy}

For centuries it has been known that when people suffer vision loss they often experience differing degrees of visual hallucinations (Ffytche, 2009; Schadlu et al., 2009). Some of these hallucinations, described as scintillations or phosphenes, which appear as spontaneous flashes or flickering of light, may originate in the retina (Lepore, 1990; Murtha and Stasheff, 2003). The first physiological evidence that spontaneous activity during retinal degeneration (rd) arises in the retina was found in recordings from neurons in the visual system of a mouse model for rd (Dräger and Hubel, 1978). In this study, it was found that rd was associated with rhythmic activity in neurons in visual cortex and superior colliculus. Upon asphyxiating the eye, it was found that the spontaneous activity disappeared, thus suggesting that the spurious activity was arising in the retina (Dräger and Hubel, 1978). Similar findings of increased spontaneous activity were subsequently reported in superior colliculus recordings from rd rats (Sauvé et al., 2001) and from a different rd mouse model (Ivanova et al., 2015). Physiological recordings in the rd retina have directly revealed an increase in spontaneous spike activity in ganglion cells, despite a reduction or absence of sensory input $(\mathrm{Pu}$ et al., 2006; Stasheff, 2008).

The finding of increased spontaneous activity in ganglion cells in retinae with degenerating photoreceptors has now been repeated by many labs and in several different animal strains including rd1 mice (rd caused by a mutation in the Pde6b gene; Margolis et al., 2008; Stasheff, 2008; Borowska et al., 2011; Menzler and Zeck, 2011), rd10 mice (rd caused by a mutation in the Pde6b gene; Goo et al., 2011; Stasheff et al., 2011; Toychiev et al., 2013; Biswas et al., 2014), P23H-1 rats (rd caused by a mutation in the rhodopsin gene; Sekirnjak et al., 2011), 
and Royal College of Surgeons (RCS) rats (rd caused by a mutation in the Mertk gene; $\mathrm{Pu}$ et al., 2006). While increased spiking is observed in ganglion cells in all models, the details regarding the precise timing of photoreceptor degeneration and whether $\mathrm{ON}$ or OFF ganglion cell circuits are more affected appears to vary between lines. Here, we focus on experiments performed primarily in fast onset retinal degeneration ( rd1) and slow onset retinal degeneration ( $\mathrm{rd} 10)$ mice, since the majority of the work examining the biophysical underpinnings of this spontaneous retinal activity has been undertaken using these lines.

One of the most noticeable features of the spontaneous activity in the $\operatorname{rd} 1$ and $\operatorname{rd} 10$ retina is that there is a tendency for ganglion cells to fire rhythmically, at a frequency of $\sim 10 \mathrm{~Hz}$ (Margolis et al., 2008; Stasheff, 2008; Borowska et al., 2011; Menzler and Zeck, 2011; Stasheff et al., 2011; Yee et al., 2012; Biswas et al., 2014). Such spontaneous activity could impair strategies that attempt to restore sight to blind retinae. Indeed it has been shown that this neural noise impairs the fidelity of synaptic transmission to ganglion cells when bipolar cells are ectopically electrically stimulated (Yee et al., 2012). In order to better understand the spontaneous activity and apply this knowledge to the design of vision restoration strategies, it is important to address two central questions: in which cells is this spontaneous activity arising and what are the biophysical properties that underlie it?

\section{Spontaneous Activity Arises at the Level of the All Amacrine/ON Cone Bipolar Cell Network}

One possibility is that structural changes and rewiring of retinal circuits may lead to spontaneous activity during photoreceptor degeneration. While gross anatomical changes have been noted in the outer retina in several rd models (Strettoi and Pignatelli, 2000; Strettoi et al., 2002; Jones et al., 2003), only minor morphological changes have been shown to occur in ganglion cells during rd (Mazzoni et al., 2008; Damiani et al., 2012; Lin and Peng, 2013; O'Brien et al., 2014). In rd mice, even after most photoreceptors have degenerated, ganglion cells can still be categorized as either ON, OFF or ON-OFF, based on their stereotypical dendritic stratification patterns in the innerplexiform layer (Margolis et al., 2008; Mazzoni et al., 2008; Borowska et al., 2011; Damiani et al., 2012; Yee et al., 2012, 2014; Lin and Peng, 2013; O'Brien et al., 2014). These findings, together with the physiological results discussed below, indicate that gross anatomical rewiring of the retinal circuitry is not likely to underlie the emergence of spontaneous activity in the degenerating retina.

Several studies combining electrophysiological and pharmacological approaches suggest that a physiological remodeling of the inner retina appears to give rise to spontaneous activity in ganglion cells. Whole-cell voltage-clamp recordings have revealed that ganglion cells receive spontaneous excitatory and inhibitory oscillatory inputs in rd retinae (Margolis et al., 2008; Borowska et al., 2011; Yee et al., 2012, 2014). When glutamatergic chemical synaptic input to ganglion cells is pharmacologically blocked, spontaneous oscillatory ganglion cell spiking is largely inhibited (Menzler and Zeck, 2011). Therefore, a major source of spontaneous activity in the rd retina must be presynaptic to retinal ganglion cells.

An initial hypothesis was that certain inhibitory amacrine cells, such as wide-field or starburst amacrine cells, which have been shown to exhibit rhythmogenic behavior in wild type retinae (Solessio et al., 2002; Vigh et al., 2003; Petit-Jacques et al., 2005), might drive oscillatory activity in the rd retina (Margolis and Detwiler, 2011; Yee et al., 2012). In this model, activity in amacrine cells would be conveyed to ganglion cells via modulation of glutamate release from bipolar cell axon terminals. Pharmacological assessment of spontaneous activity in retinal ganglion cells, however, did not support this hypothesis. Blocking inhibition was found to increase spontaneous ganglion cell activity, suggesting that spontaneous ganglion cell spiking is primarily driven by excitatory input from presynaptic bipolar cells (Borowska et al., 2011; Menzler and Zeck, 2011). However, this issue was not clear cut as some spontaneous inhibitory inputs to ganglion cells persisted in the presence of glutamate receptor blockers (Borowska et al., 2011; Menzler and Zeck, 2011). While these inhibitory inputs alone were ineffective in driving significant rhythmic output from retinal ganglion cells (Menzler and Zeck, 2011), these findings suggested that an upstream element was active in the absence of glutamatergic signaling.

Which cells presynaptic to ganglion cells are generating the spontaneous activity? Whole-cell recordings were subsequently obtained from bipolar and amacrine cells in the whole mount rd retina. These recordings revealed membrane oscillations, with a frequency of $\sim 10 \mathrm{~Hz}$, in AII amacrine and cone bipolar cells (Borowska et al., 2011; Trenholm et al., 2012) that were similar to those observed in ganglion cells. Applying a cocktail of excitatory and inhibitory chemical synaptic blockers did not inhibit oscillations in AII amacrine or ON cone bipolar cells (Borowska et al., 2011), providing the first evidence of presynaptic intrinsic oscillatory elements. Interestingly, oscillations in AII amacrine cells in rd retinae have also been observed in 200-300 $\mu \mathrm{m}$ thick retinal slices (Choi et al., 2014; Margolis et al., 2014), indicating that a reduced network is sufficient to drive neural oscillations.

In the wild type retina, AII amacrine cells are strongly coupled to the axon terminals of ON cone bipolar cells as well as to other AII amacrine cells (Famiglietti and Kolb, 1975; Strettoi et al., 1992, 1994; Veruki and Hartveit, 2002a,b). In addition, AII amacrine cells also provide glycinergic inhibition to axon terminals of OFF cone bipolar cells and dendrites of OFF ganglion cells (Pourcho and Goebel, 1985; Ivanova et al., 2006; Münch et al., 2009). Thus, if spontaneous ganglion cell activity originates in the AII amacrine/ON cone bipolar cell network, activity would be expected to occur out of phase in neighboring $\mathrm{ON}$ and OFF ganglion cells (Kerschensteiner and Wong, 2008). This prediction was recently confirmed by simultaneous measurements of spontaneous activity from neighboring $\mathrm{ON}$ and OFF ganglion cells in rd retinae (Margolis et al., 2014; Menzler et al., 2014). Additionally, oscillatory responses of OFF, but not $\mathrm{ON}$, retinal ganglion cells in rd retinae were antagonized when glycinergic synaptic transmission was pharmacologically blocked 
(Poria and Dhingra, 2015). Thus, there appears to be a general agreement that the AII amacrine/ON cone bipolar cell network is responsible for pacemaker activity in the degenerating retina.

Are other cell types spontaneously active in the degenerating retina? Certain ganglion cells appear to have slightly higher spontaneous firing rates in synaptic blockers in rd retina compared to wild type retina (Sekirnjak et al., 2011), though the mechanism behind this increased activity is unknown. Next, recent calcium imaging studies have found spontaneous rhythmic activity in the outer retina, including in the remaining cell bodies of degenerated photoreceptors and in horizontal cells (Haq et al., 2014). The frequency of the rhythmic spontaneous activity in the outer retina was slower $(\sim 3 \mathrm{~Hz})$ than the activity previously described in AII amacrine, cone bipolar, and ganglion cells $(\sim 10 \mathrm{~Hz})$. Therefore while the spontaneous activity in the outer retina may modulate activity to some extent in the degenerating retina, the key pacemaker lies at the level of AII amacrine/ON cone bipolar cells. This pacemaker activity can then diversify in different ganglion cell types (Sanes and Masland, 2015) when different presynaptic microcircuits are activated (Yee et al., 2014).

\section{TTX-Sensitive $\mathrm{Na}^{+}$Channels and Gap Junction Mediated Network Interactions Underlie Pacemaker Activity}

To gain an understanding of the biophysical basis for oscillations in the AII amacrine/ON cone bipolar cell network in the rd retina, previous studies have largely relied on pharmacology (see Figure 1 for a summary). Some of the first candidates for driving oscillations were voltage-gated $\mathrm{Ca}^{2+}$ channels in bipolar cells, as these have been shown to drive regenerative rhythmic activity in isolated cells (Burrone and Lagnado, 1997; Ma and Pan, 2003; Palmer, 2006). However, since oscillations in AII amacrine cells and $\mathrm{ON}$ cone bipolar cells in rd retinae were not blocked upon application of voltage-gated $\mathrm{Ca}^{2+}$ channel blockers (Borowska et al., 2011), these bipolar cell conductances cannot be fundamental in driving oscillations in rd retinae. Another possible candidate was the hyperpolarization activated currents $\left(\mathrm{I}_{\mathrm{h}}\right)$, which mediate oscillatory activity in other parts of the brain (Gauss and Seifert, 2000). $\mathrm{I}_{\mathrm{h}}$ is prominent in many cone bipolar cells, but not in AII amacrine cells (Trenholm et al., 2012). Blocking $\mathrm{I}_{\mathrm{h}}$ leads to membrane hyperpolarization of the AII amacrine/ON cone bipolar cell network (indicating $\mathrm{I}_{\mathrm{h}}$ is active during ongoing activity), an increase in oscillation amplitude and a decrease in oscillation frequency (Figure 1A; Trenholm et al., 2012). Thus while active conductances in bipolar cell terminals can strongly modulate the amplitude and frequency of spontaneous oscillations in rd retinae, they do not appear to generate oscillations.

In contrast to $\mathrm{I}_{\mathrm{h}}$ and voltage-gated $\mathrm{Ca}^{2+}$ channels, blocking voltage-gated $\mathrm{Na}^{+}$channels with Tetrodotoxin (TTX) completely abolishes oscillatory synaptic input to retinal ganglion cells (Trenholm et al., 2012), as well as completely blocks spontaneous oscillations in both AII amacrine and ON cone bipolar cells recorded in the whole mount retina

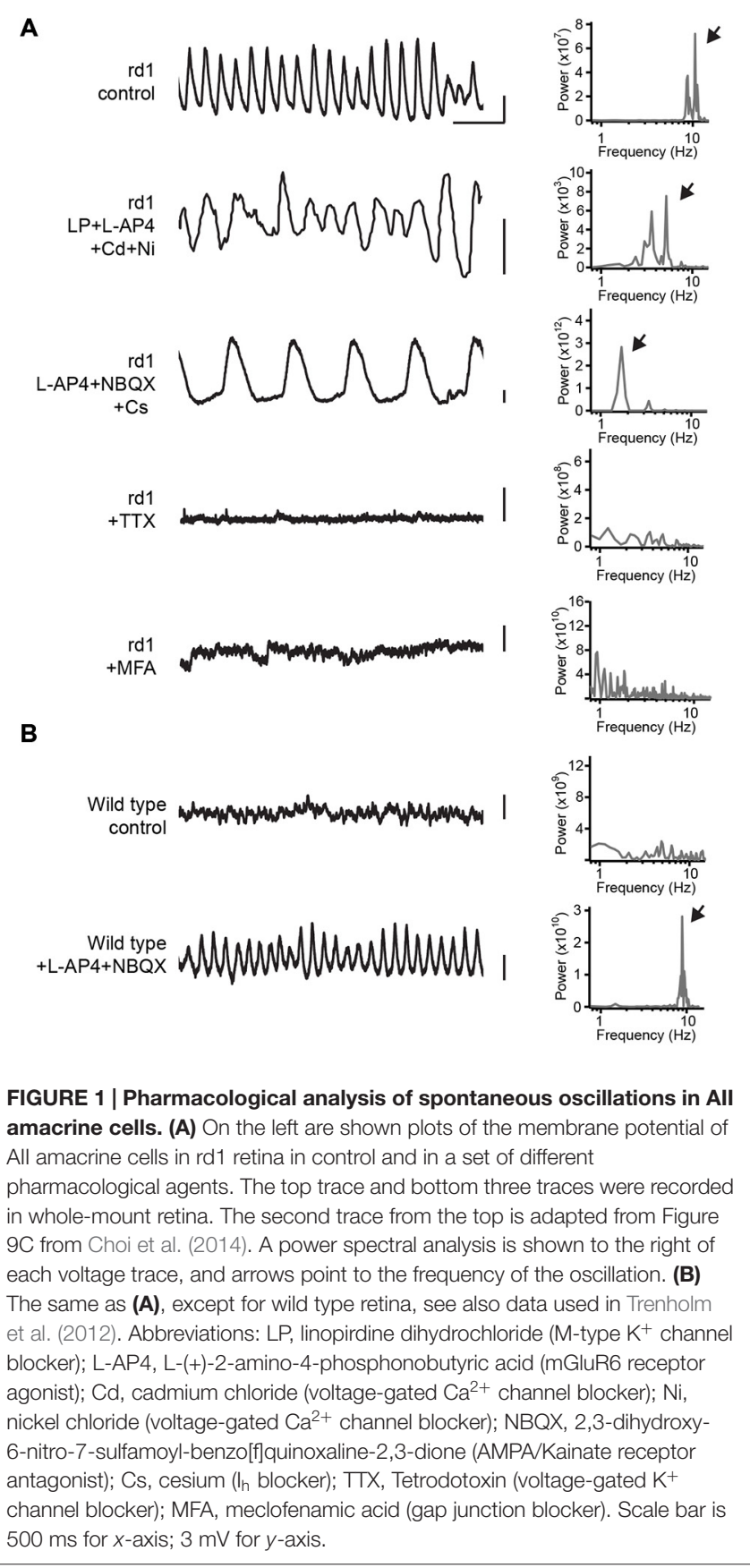

(Figure 1A; Trenholm et al., 2012) and in slice preparation (Choi et al., 2014; Margolis et al., 2014). Since voltage-gated $\mathrm{Na}^{+}$ channels are strongly expressed in AII amacrine cells where they drive spiking responses, but are only weakly expressed by a few bipolar cell types in mouse retina (Pan and Hu, 2000; Cui and Pan, 2008), given the widespread nature of oscillatory inputs to diverse ganglion cell types it is likely that $\mathrm{Na}^{+}$channels in AII amacrine cells are the primary drivers of pacemaker activity.

In addition to voltage-gated $\mathrm{Na}^{+}$channels, gap junctions have also been found to play a critical role in generating oscillations. Consistent with AII amacrine and ON cone bipolar 
cells being gap junction coupled in rd retinae (similar to what has been shown in wild type retinae, as described above), strongly modulating the membrane potential (from -80 to $0 \mathrm{mV}$ ) does not abolish spontaneous membrane oscillations (Borowska et al., 2011) and gap junction blockers significantly increase input resistance. Importantly, it was found that applying gap junction blockers eliminated membrane oscillations for both AII amacrine and ON cone bipolar cells recorded in whole mount retinae (Figure 1A; Trenholm et al., 2012) as well as for AII amacrine cells in retinal slices (Choi et al., 2014). Thus, individually, neither AII amacrine nor ON cone bipolar cells appear responsible for generating the pacemaker activity. Moreover, gap junction blockers also inhibit oscillatory activity in downstream ganglion cells (Menzler and Zeck, 2011; Trenholm et al., 2012), and knocking out connexin 36, which makes up gap junctions in many retinal cell types including AII amacrine cells, greatly reduces spontaneous input to retinal ganglion cell in rd retinae (Ivanova et al., 2015). Therefore, it appears that gap junctions play a critical role in generating oscillatory activity in the degenerating retina.

While the general biophysical framework of network oscillations has been well established, the precise mechanism whereby gap junctions enable oscillations to arise is not entirely clear. Computational modeling suggests that minor cellular heterogeneities could result in widespread network oscillations in rd retina, as has been suggested to occur in other parts of the central nervous system (CNS; Manor et al., 1997). For example, currents flowing through gap junctions would tend to equalize small differences in resting membrane potentials between neighboring AII amacrine cells and/or between ON bipolar and AII amacrine cells (Trenholm et al., 2012). Since changes in membrane potential can lead to the recruitment of the non-linear elements of individual neurons, in such a situation the membrane potential never settles and thus the network oscillates. In such a model oscillations are an emergent property of the gap junction coupled network (Manor et al., 1997; Trenholm et al., 2012; Margolis et al., 2014).

In contrast to the abovementioned network model for oscillations, a recent study has argued that oscillations in the rd retina arise from intrinsic bursting of individual AII amacrine cells, mediated by an interplay between voltage-gated $\mathrm{Na}^{+}$and M-type $\mathrm{K}^{+}$channels (Choi et al., 2014). This study suggests that the primary role for gap junctions is to allow relatively depolarized ON cone bipolar cells to depolarize AII amacrine cells and bring their membrane potential into a state that promotes intrinsic oscillations (Choi et al., 2014). However, while M-type $\mathrm{K}^{+}$channels clearly are important in controlling the kinetics of spontaneous oscillations, the model presented by Choi et al. (2014) is not completely supported by their data: (1) In the rd retina, in the presence of gap junction blockers, AII amacrine cells do not appear to sustain a regular $\sim 10 \mathrm{~Hz}$ oscillation when depolarized (compare Figures 6A,B in Choi et al., 2014); (2) Oscillations in AII amacrine cells persist when M-type $\mathrm{K}^{+}$ channels are blocked [even in the added presence of voltagegated $\mathrm{Ca}^{2+}$ channel blockers (see Figure 1A; adapted from Figure 9C in Choi et al., 2014)]; (3) Pharmacologically activating $\mathrm{M}$-type $\mathrm{K}^{+}$channels does not block membrane oscillations in ganglion cells in rd retinae (see Figure 10A in Choi et al., 2014). Nonetheless, it should be noted that a limitation of these studies is that they rely on pharmacology to demonstrate the importance of gap junctions in driving network oscillations by indiscriminately blocking gap junctions between AII amacrine cells and other AII amacrine cells, as well as between AII amacrine cells and ON cone bipolar cells. In addition, gap junction blockers can have non-specific effects. For instance, it appears that the widely used gap junction blocker meclofenamic acid can directly activate M-type $\mathrm{K}^{+}$channels (Peretz et al., 2005). Future studies using cell-specific knock out of gap junctions (either exclusively from AII amacrine or ON cone bipolar cells) will help to elucidate precisely how gap junctions in the AII amacrine/ON cone bipolar cell network promote oscillations.

Finally, if TTX-sensitive $\mathrm{Na}^{+}$channels are present in AII amacrine cells in wild type retinae, and AII amacrine cells are extensively coupled to each other as well as to ON cone bipolar cells in wild type retinae, why do these components only contribute to the generation of network wide oscillations in rd retinae? Critical insights into the mechanism underlying oscillations came from pharmacological experiments in wild type retinae showing that hyperpolarizing the AII amacrine/ON cone bipolar cell network by pharmacologically blocking photoreceptor output led to the emergence of $\sim 10 \mathrm{~Hz}$ oscillations in wild type AII amacrine/ON cone bipolar cells (Figure 1B; Trenholm et al., 2012). These pharmacologically induced oscillations exhibited the same pharmacological properties as oscillations in the rd retina (Trenholm et al., 2012), suggesting a common underlying mechanism. Similar results have also been found when wild type retinae were bleached with light (Menzler et al., 2014). These finding suggests that no overt changes in the biophysical properties of the AII amacrine/ON cone bipolar cell network are required upon rd to drive oscillations. Instead, it appears that a network hyperpolarization-resulting from the loss of photoreceptor input to bipolar cells-sets the AII amacrine/ON cone bipolar cell network into an oscillatory state. Indeed, direct measurements of the resting potential of AII amacrine cells in the rd retina have revealed that they are more hyperpolarized compared to their wild type counterparts (Choi et al., 2014). Membrane hyperpolarization of the AII amacrine/ON cone bipolar cell network may serve to increase the pool of available $\mathrm{Na}^{+}$channels by bringing them out of an inactivated state, and thereby induce oscillations (Trenholm et al., 2012). Thus, dramatic changes in ganglion cell spike activity during $r d$ appear to arise from relatively moderate changes in the steady-state membrane potential of presynaptic neurons.

\section{Summary}

Taken together, these experiments reveal several key features of the retina following photoreceptor degeneration and these are outlined in Figure 2. First, spontaneous activity increases after the loss of photoreceptor input and leads to rhythmic activity in retinal ganglion cells. The primary source of this spontaneous noise lies in the electrically coupled network of AII amacrine and ON cone bipolar cells. This coupled network appears to become spontaneously active partly due to a 
A

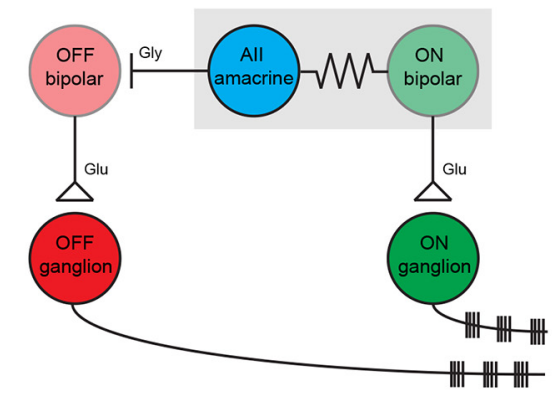

FIGURE 2 | The simplified circuit diagram for spontaneous oscillations in the rd retina. (A) A simplified circuit showing the major neuronal cell types that play a role in generating oscillations in the rd retina (adapted from Borowska et al., 2011; Margolis et al., 2014). The oscillation is an intrinsic property of the electrically coupled network of All amacrine cells and ON cone bipolar cells (indicated with the gray box). All amacrine cells inhibit OFF bipolar
B

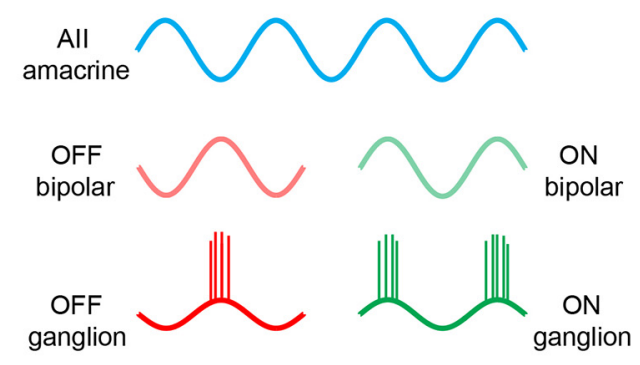

cells with glycine (labeled as gly). ON and OFF bipolar cells activate $\mathrm{ON}$ and OFF ganglion cells, respectively, via glutamate release (labeled as glu). Neighboring ON and OFF ganglion cells oscillate out of phase with one another (B). Oscillation in the All amacrine/ON cone bipolar cell network interact with multiple types of amacrine cells and result in altered dynamics in the $\sim 30$ types of ganglion cell microcircuits (not shown). small hyperpolarization that occurs upon loss of photoreceptor input during $\mathrm{rd}$. Second, blocking gap junctions inhibits oscillations, meaning that oscillations arise from cross-junctional interactions of conductances between different neurons in the AII amacrine/ON cone bipolar cell network. Third, voltagegated $\mathrm{Na}^{+}$channels are required for driving the oscillation. Fourth, bipolar cells appear to play a key role in setting the membrane potential of the coupled network into a state in which it can oscillate, and active membrane properties of bipolar cells, including $I_{h}$, regulate the amplitude and frequency of oscillations. Finally, no major rewiring of the retina appears to be required for driving oscillations, as pharmacologically blocking photoreceptor output or photo-bleaching the wild type retina generates similar pacemaker activity. Future work targeting the different components that modulate this spontaneous activity may allow for this spontaneous noise to be dampened in order to

\section{References}

Biswas, S., Haselier, C., Mataruga, A., Thumann, G., Walter, P., and Müller, F. (2014). Pharmacological analysis of intrinsic neuronal oscillations in rd10 retina. PloS One 9:e99075. doi: 10.1371/journal.pone. 0099075

Borowska, J., Trenholm, S., and Awatramani, G. B. (2011). An intrinsic neural oscillator in the degenerating mouse retina. J. Neurosci. 31, 5000-5012. doi: 10. 1523/JNEUROSCI.5800-10.2011

Burrone, J., and Lagnado, L. (1997). Electrical resonance and Ca2+ influx in the synaptic terminal of depolarizing bipolar cells from the goldfish retina. J. Physiol. 505, 571-584. doi: 10.1111/j.1469-7793.1997.571ba.x

Choi, H., Zhang, L., Cembrowski, M. S., Sabottke, C. F., Markowitz, A. L., Butts, D. A., et al. (2014). Intrinsic bursting of AII amacrine cells underlies oscillations in the rd1 mouse retina. J. Neurophysiol. 112, 1491-1504. doi: 10.1152/jn.00437. 2014

Cui, J., and Pan, Z.-H. (2008). Two types of cone bipolar cells express voltagegated $\mathrm{Na}+$ channels in the rat retina. Vis. Neurosci. 25, 635-645. doi: 10. $1017 / \mathrm{s} 0952523808080851$

Damiani, D., Novelli, E., Mazzoni, F., and Strettoi, E. (2012). Undersized dendritic arborizations in retinal ganglion cells of the rd1 mutant mouse: a paradigm of early onset photoreceptor degeneration. J. Comp. Neurol. 520, 1406-1423. doi: $10.1002 /$ cne. 22802 heighten the effectiveness of vision restoration strategies. Indeed, such work has already commenced (Toychiev et al., 2013). Additionally, performing similar experiments in vivo, such as injecting pharmacological agents into the eye while monitoring spontaneous activity in higher visual centers, will be important for outlining which strategies for mitigating spontaneous retinal activity have the best chance of translating from the lab to the clinic.

\section{Acknowledgments}

Rights were obtained for the use of Figure 9C from Choi et al. (2014) (license number 3660890219116). We acknowledge the following funding sources: Human Frontier Science Program Postdoctoral Fellowship (LT000173/2013) to ST and Foundation Fighting Blindness to GBA.

Dräger, U. C., and Hubel, D. H. (1978). Studies of visual function and its decay in mice with hereditary retinal degeneration. J. Comp. Neurol. 180, 85-114. doi: 10.1002/cne.901800107

Famiglietti, E. V. Jr., and Kolb, H. (1975). A bistratified amacrine cell and synaptic cirucitry in the inner plexiform layer of the retina. Brain Res. 84, 293-300. doi: 10.1016/0006-8993(75)90983-x

Ffytche, D. H. (2009). Visual hallucinations in eye disease. Curr. Opin. Neurol. 22, 28-35. doi: 10.1097/wco.0b013e32831f1b3f

Gauss, R., and Seifert, R. (2000). Pacemaker oscillations in heart and brain: a key role for hyperpolarization-activated cation channels. Chronobiol. Int. 17, 453-469. doi: 10.1081/cbi-100101057

Goo, Y. S., Ahn, K. N., Song, Y. J., Ahn, S. H., Han, S. K., Ryu, S. B., et al. (2011). Spontaneous Oscillatory Rhythm in Retinal Activities of Two Retinal Degeneration (rd1 and rd10) Mice. Korean J. Physiol. Pharmacol. 15, 415-422. doi: 10.4196/kjpp.2011.15.6.415

Haq, W., Arango-Gonzalez, B., Zrenner, E., Euler, T., and Schubert, T. (2014) Synaptic remodeling generates synchronous oscillations in the degenerated outer mouse retina. Front. Neural Circuits 8:108. doi: 10.3389/fncir.2014.00108

Ivanova, E., Müller, U., and Wässle, H. (2006). Characterization of the glycinergic input to bipolar cells of the mouse retina. Eur. J. Neurosci. 23, 350-364. doi: 10. 1111/j.1460-9568.2005.04557.x

Ivanova, E., Yee, C. W., Baldoni, R. Jr., and Sagdullaev, B. T. (2015). Aberrant activity in retinal degeneration impairs central visual processing and relies on 
Cx36-containing gap junctions. Exp. Eye Res. doi: 10.1016/j.exer.2015.05.013 [Epub ahead of print].

Jones, B. W., Watt, C. B., Frederick, J. M., Baehr, W., Chen, C.K., Levine, E. M., et al. (2003). Retinal remodeling triggered by photoreceptor degenerations. J. Comp. Neurol. 464, 1-16. doi: 10.1002/cne. 10703

Kerschensteiner, D., and Wong, R. O. L. (2008). A precisely timed asynchronous pattern of $\mathrm{ON}$ and $\mathrm{OFF}$ retinal ganglion cell activity during propagation of retinal waves. Neuron 58, 851-858. doi: 10.1016/j.neuron.2008. 04.025

Lepore, F. E. (1990). Spontaneous visual phenomena with visual loss: 104 patients with lesions of retinal and neural afferent pathways. Neurology 40, 444-447. doi: 10.1212/wnl.40.3_part_1.444

Lin, B., and Peng, E. B. (2013). Retinal ganglion cells are resistant to photoreceptor loss in retinal degeneration. PloS One 8:e68084. doi: 10.1371/journal.pone. 0068084

Ma, Y.-P., and Pan, Z.-H. (2003). Spontaneous regenerative activity in mammalian retinal bipolar cells: roles of multiple subtypes of voltage-dependent $\mathrm{Ca} 2+$ channels. Vis. Neurosci. 20, 131-139. doi: 10.1017/s0952523803202042

Manor, Y., Rinzel, J., Segev, I., and Yarom, Y. (1997). Low-amplitude oscillations in the inferior olive: a model based on electrical coupling of neurons with heterogeneous channel densities. J. Neurophysiol. 77, 2736-2752.

Margolis, D. J., and Detwiler, P. B. (2011). Cellular origin of spontaneous ganglion cell spike activity in animal models of retinitis pigmentosa. J. Ophthalmol. 2011, 1-6. doi: 10.1155/2011/507037

Margolis, D. J., Gartland, A. J., Singer, J. H., and Detwiler, P. B. (2014). Network oscillations drive correlated spiking of $\mathrm{ON}$ and OFF ganglion cells in the rd1 mouse model of retinal degeneration. PLOS ONE 9:e86253. doi: 10. 1371/journal.pone.0086253

Margolis, D. J., Newkirk, G., Euler, T., and Detwiler, P. B. (2008). Functional stability of retinal ganglion cells after degeneration-induced changes in synaptic input. J. Neurosci. 28, 6526-6536. doi: 10.1523/JNEUROSCI.1533-08.2008

Mazzoni, F., Novelli, E., and Strettoi, E. (2008). Retinal ganglion cells survive and maintain normal dendritic morphology in a mouse model of inherited photoreceptor degeneration. J. Neurosci. 28, 14282-14292. doi: 10. 1523/JNEUROSCI.4968-08.2008

Menzler, J., Channappa, L., and Zeck, G. (2014). Rhythmic ganglion cell activity in bleached and blind adult mouse retinas. PloS One 9:e106047. doi: 10. 1371/journal.pone.0106047

Menzler, J., and Zeck, G. (2011). Network oscillations in rod-degenerated mouse retinas. J. Neurosci. 31, 2280-2291. doi: 10.1523/JNEUROSCI.4238-10.2011

Münch, T. A., da Silveira, R. A., Siegert, S., Viney, T. J., Awatramani, G. B., and Roska, B. (2009). Approach sensitivity in the retina processed by a multifunctional neural circuit. Nat. Neurosci. 12, 1308-1316. doi: 10.1038/nn. 2389

Murtha, T., and Stasheff, S. F. (2003). Visual dysfunction in retinal and optic nerve disease. Neurol. Clin. 21, 445-481. doi: 10.1016/s0733-8619(02)00108-1

O'Brien, E. E., Greferath, U., and Fletcher, E. L. (2014). The effect of photoreceptor degeneration on ganglion cell morphology. J. Comp. Neurol. 522, 1155-1170. doi: $10.1002 /$ cne. 23487

Palmer, M. J. (2006). Modulation of Ca2+-activated $\mathrm{K}+$ currents and Ca2+dependent action potentials by exocytosis in goldfish bipolar cell terminals. J. Physiol. 572, 747-762. doi: 10.1113/jphysiol.2006.105205

Pan, Z. H., and Hu, H. J. (2000). Voltage-dependent $\mathrm{Na}^{+}$currents in mammalian retinal cone bipolar cells. J. Neurophysiol. 84, 2564-2571.

Peretz, A., Degani, N., Nachman, R., Uziyel, Y., Gibor, G., Shabat, D., et al. (2005). Meclofenamic Acid and Diclofenac, Novel Templates of KCNQ2/Q3 Potassium Channel Openers, Depress Cortical Neuron Activity and Exhibit Anticonvulsant Properties. Mol. Pharmacol. 67, 1053-1066. doi: 10.1124/mol. 104.007112

Petit-Jacques, J., Völgyi, B., Rudy, B., and Bloomfield, S. (2005). Spontaneous oscillatory activity of starburst amacrine cells in the mouse retina. J. Neurophysiol. 94, 1770-1780. doi: 10.1152/jn.00279.2005

Poria, D., and Dhingra, N. K. (2015). Spontaneous oscillatory activity in rd1 mouse retina is transferred from ON pathway to OFF pathway via glycinergic synapse. J. Neurophysiol. 113, 420-425. doi: 10.1152/jn. 00702.2014
Pourcho, R. G., and Goebel, D. J. (1985). A combined Golgi and autoradiographic study of $(3 \mathrm{H})$ glycine-accumulating amacrine cells in the cat retina. J. Comp. Neurol. 233, 473-480. doi: 10.1002/cne.9023 30406

$\mathrm{Pu}, \mathrm{M}$., Xu, L., and Zhang, H. (2006). Visual response properties of retinal ganglion cells in the royal college of surgeons dystrophic rat. Invest. Ophthalmol. Vis. Sci. 47, 3579-3585. doi: 10.1167/iovs.05-1450

Sanes, J. R., and Masland, R. H. (2015). The Types of Retinal Ganglion Cells: Current Status and Implications for Neuronal Classification. Annu. Rev. Neurosci. doi: 10.1146/annurev-neuro-071714-034120 [Epub ahead of print].

Sauvé, Y., Girman, S. V., Wang, S., Lawrence, J. M., and Lund, R. D. (2001). Progressive visual sensitivity loss in the Royal College of Surgeons rat: perimetric study in the superior colliculus. Neuroscience 103, 51-63. doi: 10 . 1016/s0306-4522(00)00557-1

Schadlu, A. P., Schadlu, R., and Shepherd, J. B. IIIrd (2009). Charles Bonnet syndrome: a review. Curr. Opin. Ophthalmol. 20, 219-222. doi: 10.1097/ICU. 0b013e328329b643

Sekirnjak, C., Jepson, L. H., Hottowy, P., Sher, A., Dabrowski, W., Litke, A. M., et al. (2011). Changes in physiological properties of rat ganglion cells during retinal degeneration. J. Neurophysiol. 105, 2560-2571. doi: 10.1152/jn.01061. 2010

Solessio, E., Vigh, J., Cuenca, N., Rapp, K., and Lasater, E. M. (2002). Membrane properties of an unusual intrinsically oscillating, wide-field teleost retinal amacrine cell. J. Physiol. 544, 831-847. doi: 10.1113/jphysiol.2002. 021899

Stasheff, S. F. (2008). Emergence of sustained spontaneous hyperactivity and temporary preservation of OFF responses in ganglion cells of the retinal degeneration (rd1) mouse. J. Neurophysiol. 99, 1408-1421. doi: 10.1152/jn. 00144.2007

Stasheff, S. F., Shankar, M., and Andrews, M. P. (2011). Developmental time course distinguishes changes in spontaneous and light-evoked retinal ganglion cell activity in rd1 and rd10 mice. J. Neurophysiol. 105, 3002-3009. doi: 10.1152/jn. 00704.2010

Strettoi, E., Dacheux, R. F., and Raviola, E. (1994). Cone bipolar cells as interneurons in the rod pathway of the rabbit retina. J. Comp. Neurol. 347, 139-149. doi: 10.1002/cne.903470111

Strettoi, E., and Pignatelli, V. (2000). Modifications of retinal neurons in a mouse model of retinitis pigmentosa. Proc. Natl. Acad. Sci. U S A 97, 11020-11025. doi: 10.1073/pnas.190291097

Strettoi, E., Porciatti, V., Falsini, B., Pignatelli, V., and Rossi, C. (2002). Morphological and Functional Abnormalities in the Inner Retina of the $\mathrm{rd} / \mathrm{rd}$ Mouse. J. Neurosci. 22, 5492-5504.

Strettoi, E., Raviola, E., and Dacheux, R. F. (1992). Synaptic connections of the narrow-field, bistratified rod amacrine cell (AII) in the rabbit retina. J. Comp. Neurol. 325, 152-168. doi: 10.1002/cne.903250203

Toychiev, A. H., Ivanova, E., Yee, C. W., and Sagdullaev, B. T. (2013). Block of gap junctions eliminates aberrant activity and restores light responses during retinal degeneration. J. Neurosci. 33, 13972-13977. doi: 10.1523/JNEUROSCI. 2399-13.2013

Trenholm, S., Borowska, J., Zhang, J., Hoggarth, A., Johnson, K., Barnes, S., et al. (2012). Intrinsic oscillatory activity arising within the electrically coupled AII amacrine-ON cone bipolar cell network is driven by voltagegated Na+ channels. J. Physiol. 590, 2501-2517. doi: 10.1113/jphysiol.2011. 225060

Veruki, M. L., and Hartveit, E. (2002a). AII (Rod) amacrine cells form a network of electrically coupled interneurons in the mammalian retina. Neuron 33, 935-946. doi: 10.1016/s0896-6273(02)00609-8

Veruki, M. L., and Hartveit, E. (2002b). Electrical synapses mediate signal transmission in the rod pathway of the mammalian retina. J. Neurosci. 22, $10558-10566$.

Vigh, J., Solessio, E., Morgans, C. W., and Lasater, E. M. (2003). Ionic mechanisms mediating oscillatory membrane potentials in wide-field retinal amacrine cells. J. Neurophysiol. 90, 431-443. doi: 10.1152/jn.00092.2003

Yee, C. W., Toychiev, A. H., Ivanova, E., and Sagdullaev, B. T. (2014). Aberrant synaptic input to retinal ganglion cells varies with morphology in a mouse model of retinal degeneration. J. Comp. Neurol. 522, 4085-4099. doi: 10. $1002 /$ cne. 23660 
Yee, C. W., Toychiev, A. H., and Sagdullaev, B. T. (2012). Network deficiency exacerbates impairment in a mouse model of retinal degeneration. Front. Syst. Neurosci. 6:8. doi: 10.3389/fnsys.2012.00008

Conflict of Interest Statement: The authors declare that the research was conducted in the absence of any commercial or financial relationships that could be construed as a potential conflict of interest.
Copyright (c) 2015 Trenholm and Awatramani. This is an open-access article distributed under the terms of the Creative Commons Attribution License (CC $B Y)$. The use, distribution and reproduction in other forums is permitted, provided the original author(s) or licensor are credited and that the original publication in this journal is cited, in accordance with accepted academic practice. No use, distribution or reproduction is permitted which does not comply with these terms. 